\title{
An Introduction to the Density Matrix Renormalization Group Ansatz in Quantum Chemistry
}

\author{
Garnet Kin-Lic Chan, Jonathan J. Dorando, Debashree Ghosh, Johannes \\ Hachmann, Eric Neuscamman, Haitao Wang, and Takeshi Yanai
}

\section{Introduction}

The Density Matrix Renormalization Group (DMRG) is an electronic structure method that has recently been applied to ab-initio quantum chemistry. The method originated in the condensed matter community with the pioneering work of White [1, 2]. Although the earliest quantum chemistry implementations are only a few years old, the DMRG has already been used to solve many problems that would have been intractable with any other method, and especially, multireference problems with very large active spaces. For example, we have used the DMRG to study systems ranging from molecular potential energy curves [3, 4], to excited states of large conjugated polymers [5, 6], to metal-insulator type transitions in hydrogen chains [7]. In each case, we have obtained accuracies close to the (estimated) exact Complete Active Space Configuration Interaction (CASCI) or Complete Active Space Self-Consistent-Field (CASSCF) result, for active spaces well outside the range of traditional algorithms e.g. 100 active electrons in 100 active orbitals [7]. Unlike a traditional CAS (where the active space wavefunction is obtained in a brute-force Full Configuration Interaction expansion) the DMRG utilises a compact wavefunction ansatz. However, this ansatz is very flexible, is well-suited to nondynamic correlation, and in the cases of long molecules, provides a near optimal, local description of multireference correlations.

Garnet Kin-Lic Chan, Jonathan J. Dorando, Debashree Ghosh, Johannes Hachmann, Eric Neuscamman, and Haitao Wang

Department of Chemistry and Chemical Biology, Cornell University, Ithaca, New York 148531301, USA.

Takeshi Yanai

Department of Theoretical and Computational Molecular Science, Institute for Molecular Science, Okazaki, Aichi 444-8585, Japan.

Corresponding author: Garnet Kin-Lic Chan, e-mail: gc238@cornell.edu 
Historically, the DMRG was not originally formulated from a wavefunction ansatz perspective, but rather in the Renormalisation Group (RG) language of Wilson's Numerical RG [8, 9, 1, 2], from which it is descended. The original quantum chemical implementations of the DMRG were also described from an RG point of view (e.g. [10, 11, 12, 13, 14]). Although the mathematical form of the DMRG ansatz has been known for some time [15, 16, 17, 18], only in recent years has it been realised that the wavefunction view of the DMRG provides a more convenient and in many cases more powerful paradigm, and this has led to fundamental advances in the DMRG method itself [19, 20, 21, 22, 23, 24, 25, 26, 27, 28, 29, 30, 31, 32, 7].

The current article provides an expository introduction to the DMRG in quantum chemistry from the wavefunction point of view. This is complementary to earlier articles that use the RG based formulation and the first-time reader will benefit from reading such articles alongside the current one. It is not our intention to provide a comprehensive review of the DMRG method even within the restricted domain of quantum chemistry. Thus we do not pretend to survey the literature except to say at the start that the field of quantum chemical DMRG has developed through the work of White et al.[10, 33, 34], Mitrushenkov et al.[11, 35, 36], our contributions [12, 3, 37, 4, 38, 7, 6, 5], the work of Legeza, Hess et al.[13, 39, 40, 41], the work of Reiher et al. [42, 43, 44, 14], and most recently the work of Zgid and Nooijen [45]. Also related, but too numerous to cite in full here, are the developments with semi-empirical Hamiltonians; some representative early works are those in [46, 47, 48, 49, 50, 51, 52]. In addition, we mention again that the DMRG has its origins in the condensed matter community and thus excellent sources of information which provide this perspective are the recent reviews of Schollwöck [32] and Hallberg [30, 31].

The structure of our article is as follows. We begin by introducing the underlying DMRG ansatz and examining some of its special properties in sections 2 and 3 , In sections 4 and 5 we explain the connection between the wavefunction ansatz, and the original Renormalisation Group language within which the DMRG is usually described. In section 6 we describe how the structure of the DMRG wavefunction allows the efficient evaluation of Hamiltonian matrix elements. Finally, we finish with some brief thoughts and conclusions in section 7

\section{Motivation for the DMRG Ansatz}

The primary challenge in quantum chemistry is to find a good approximation to the electronic wavefunction of a quantum state. We can express any $N$-electron wavefunction in a complete basis of Slater determinants, through the Full Configuration Interaction (FCI) expansion, 


$$
\begin{aligned}
|\Psi\rangle & =\sum_{n_{1} n_{2} n_{3} \ldots n_{k}} \Psi^{n_{1} n_{2} n_{3} \ldots n_{k}}\left|n_{1} n_{2} n_{3} \ldots n_{k}\right\rangle, \\
\left\{n_{i}\right\} & =\left\{|0\rangle,\left|1^{\alpha}\right\rangle,\left|1^{\beta}\right\rangle,\left|2^{\alpha \beta}\right\rangle\right\} \\
\sum_{i} n_{i} & =N .
\end{aligned}
$$

Here $\left|n_{1} \ldots n_{k}\right\rangle$ is the occupation number representation of the Slater determinant where $n_{i}$ is the occupation of site (i.e. orbital) $i$. The total number of orbitals is $k$ and $N$ is the total number of electrons.

The dimension of the coefficient tensor $\Psi$ in the above expansion is $4^{k}$, which is intractable for values of $k$ much larger than 10 . Therefore, we would like to find an ansatz where $\Psi$ is expressed more compactly. In particular, we would want such an ansatz to require only a polynomial amount of information as a function of the number of orbitals in the system, $k$.

A very simple ansatz would be to approximate the high-dimensional coefficient tensor $\Psi$ by a tensor product of vectors $\psi^{1} \ldots \psi^{k}$, which we shall call site functions,

$$
\Psi \approx \psi^{1} \otimes \psi^{2} \otimes \psi^{3} \ldots \otimes \psi^{k} .
$$

Using the notation $\psi^{n_{1}}$ to denote the $n$th element of $\psi^{1}$, i.e. $\psi^{n_{1}}=\psi_{n}^{i}$, we can also write

$$
\Psi^{n_{1} n_{2} n_{3} \ldots n_{k}} \approx \psi^{n_{1}} \psi^{n_{2}} \psi^{n_{3}} \ldots \psi^{n_{k}}
$$

Note that each site function $\psi$ is not an orbital but rather a vector of length 4 , and $\psi^{n_{1}}, \psi^{n_{2}}$ represent elements of the different vectors $\psi^{1}, \psi^{2}$. This ansatz contains only $4 k$ parameters and is certainly tractable. However, it is also not, in general, very accurate. So, let us try to improve the ansatz by increasing the flexibility of the site functions $\psi$. We can introduce additional auxiliary indices, i.e.

$$
\psi^{n_{p}} \rightarrow \psi_{i i^{\prime}}^{n_{p}}
$$

The new indices $i, i^{\prime}$ are auxiliary in the sense that they do not appear in the final coefficient tensor $\Psi$ and must be contracted over in some fashion. The simplest arrangement is to contract the indices sequentially from one $\psi$ site function to the next, i.e.

$$
\Psi^{n_{1} n_{2} n_{3} \ldots n_{k}} \approx \sum_{i_{1} i_{2} i_{3} \ldots i_{k-1}} \psi_{i_{1}}^{n_{1}} \psi_{i_{1} i_{2}}^{n_{2}} \psi_{i_{2} i_{3}}^{n_{3}} \ldots \psi_{i_{k-1}}^{n_{k}} .
$$

For simplicity, we will assume that the dimensions of all auxiliary indices are chosen to be the same, and we shall call this dimension $M$. Then each site function $\psi$ is a 3-tensor of dimension $4 \times M \times M$, and the total number of parameters in the wavefunction ansatz is $4 M^{2} k$.

This is, in essence, the DMRG ansatz for $M$ states. (More precisely, it is the ansatz used in the one-site DMRG algorithm, as explained later). Note that by increasing the dimension $M$, we can make the approximation arbitrarily exact. Because (for given $n_{1} \ldots n_{k}$ ) the contraction in Eq. (7) is a series of matrix 
products, this ansatz is referred to in the literature as the Matrix Product State $[15,16,17,18,53,19,25,54,55]$. Combining the site functions explicitly with the Slater determinants we have

$$
\left|\Psi_{\mathrm{DMRG}}\right\rangle=\sum_{\substack{n_{1} n_{2} n_{3} \ldots n_{k} \\ i_{1} i_{2} i_{3} \ldots i_{k-1}}} \psi_{i_{1}}^{n_{1}} \psi_{i_{1} i_{2}}^{n_{2}} \psi_{i_{2} i_{3}}^{n_{3}} \ldots \psi_{i_{k-1}}^{n_{k}}\left|n_{1} n_{2} n_{3} \ldots n_{k}\right\rangle
$$

Before continuing, let us first establish some notation. The site functions $\psi$ in Eq. (8) are 3-tensors. However, the notation of linear algebra is designed primarily for vectors (1-tensors) and matrices (2-tensors). Naturally, any 3-tensor can be considered as an array of matrices, so long as we specify which two indices are the matrix indices and which is the 3rd (array) index. When viewing the site function as an array of matrices, we will write the 3rd (array) index on the top. Thus in this notation, we have

$$
\begin{aligned}
& \text { Matrix : }\left[\psi^{n_{p}}\right](\text { dimension } M \times M) \\
& \text { Elements : } \psi_{i_{p-1} i_{p}}^{n_{p}}
\end{aligned}
$$

and the DMRG wavefunction (8) is written as

$$
\left|\Psi_{\mathrm{DMRG}}\right\rangle=\sum_{n_{1} n_{2} n_{3} \ldots n_{k}}\left[\psi^{n_{1}}\right]\left[\psi^{n_{2}}\right]\left[\psi^{n_{3}}\right] \ldots\left[\psi^{n_{k}}\right]\left|n_{1} n_{2} n_{3} \ldots n_{k}\right\rangle
$$

(Note that the first and last site functions $\left[\psi^{n_{1}}\right],\left[\psi^{n_{k}}\right]$ have dimensions $1 \times M$ and $M \times 1$ respectively).

Alternatively, we can view a 3-tensor as a single matrix if we group two indices together to make a compound index. This view will be useful when discussing the renormalised basis and canonical representations of the DMRG wavefunction in sections 4 and 5 Depending on the context, we will either group the $n$ index with the left or the right auxiliary indices, giving

$$
\begin{aligned}
& \text { Matrix : }\left[\psi^{p}\right](\text { dimension } 4 M \times M) \\
& \text { Elements : } \psi_{n i, i^{\prime}}^{p} \\
& \text { or Matrix : }\left[\psi^{p}\right](\text { dimension } M \times 4 M) \\
& \text { Elements : } \psi_{i, n i^{\prime}}^{p}
\end{aligned}
$$

Note that the superscript $p$ here denotes the $p$ th site function in the DMRG ansatz (8), not any particular element of the site function.

\section{Properties of the DMRG ansatz}

Let us now examine some properties of the DMRG ansatz. 
1. Variational: Since we have an explicit wavefunction, the expectation value of the energy provides a variational upper bound to the true energy and in practice DMRG energies are evaluated in this way. As $M$ is increased, the DMRG energy converges from above to the exact energy.

2. Multireference: There is no division into occupied and virtual orbitals, all orbitals appear on an equal footing in the ansatz (8). In particular, the Hartree-Fock reference has no special significance here. For this reason, we expect (and observe) the ansatz to be very well-balanced for describing nondynamic correlation in multireference problems (see e.g. [37, 4, 7]). Conversely, the ansatz is inefficient for describing dynamic correlation, since this benefits from knowledge of the occupied and virtual spaces.

3. Size-consistency: The DMRG ansatz is size-consistent within a localised basis. Consider a system $A B$ composed of two spatially separated, non-interacting subsystems $A$ and $B$. Associate localised orbitals $1 \ldots a$ with subsystem $A$ and $a+1 \ldots a+b$ with subsystem $B$. Then, the DMRG wavefunction for $A B$ factorises into a product of DMRG wavefunctions for $A$ and $B$. First expand the DMRG wavefunction

$$
\begin{aligned}
\left|\Psi_{\mathrm{DMRG}}^{\mathrm{AB}}\right\rangle & =\sum_{\substack{n_{1} \ldots n_{a+b} \\
i_{1} \ldots i_{a+b-1}}} \psi_{i_{1}}^{n_{1}} \ldots \psi_{i_{a-1} i_{a}}^{n_{a}} \psi_{i_{a} i_{a+1}}^{n_{a+1}} \ldots \psi_{i_{a+b-1}}^{n_{a+b}}\left|n_{1} \ldots n_{a} n_{a+1} n_{a+b}\right\rangle \\
& =\sum_{i_{a}}\left(\sum_{\substack{n_{1} \ldots n_{a} \\
i_{1} \ldots i_{a-1}}} \psi_{i_{1}}^{n_{1}} \ldots \psi_{i_{a-1} i_{a}}^{n_{a}}\left|n_{1} \ldots n_{a}\right\rangle\right. \\
& \left.\times \sum_{\substack{n_{a+1} \ldots n_{a+b} \\
i_{a+1} \ldots i_{a+b-1}}} \psi_{i_{a} i_{a+1}}^{n_{a+1}} \psi_{i_{a+b-1}}^{n_{a+b}}\left|n_{a+1} \ldots n_{a+b}\right\rangle\right) .
\end{aligned}
$$

Then note that we can write a separable wavefunction $\left|\Psi^{\mathrm{AB}}\right\rangle=\left|\Psi^{\mathrm{A}}\right\rangle\left|\Psi^{\mathrm{B}}\right\rangle$ formally as $\left|\Psi^{\mathrm{AB}}\right\rangle=\sum_{i=1}^{1}\left|\Psi_{i}^{\mathrm{A}}\right\rangle\left|\Psi_{i}^{\mathrm{B}}\right\rangle$ and thus we can take the dimension of index $i_{a}$ which couples systems $A$ and $B$ above to be 1, giving

$$
\begin{aligned}
\left|\Psi_{\mathrm{DMRG}}^{\mathrm{AB}}\right\rangle & =\sum_{\substack{n_{1} \ldots n_{a} \\
i_{1} \ldots i_{a-1}}} \psi_{i_{1}}^{n_{1}} \ldots \psi_{i_{a-1}}^{n_{a}}\left|n_{1} \ldots n_{a}\right\rangle \sum_{\substack{n_{a+1} \ldots n_{a+b} \\
i_{a+1} \ldots i_{a+b}}} \psi_{i_{a+1}}^{n_{a+1}} \psi_{i_{a+b-1}}^{n_{a+b}}\left|n_{a+1} \ldots n_{a+b}\right\rangle \\
& =\left|\Psi_{\mathrm{DMRG}}^{\mathrm{A}}\right\rangle\left|\Psi_{\mathrm{DMRG}}^{\mathrm{B}}\right\rangle .
\end{aligned}
$$

4. Compactness and efficiency of the ansatz: The number of variational parameters in the DMRG ansatz is $O\left(M^{2} k\right)$. How large do we need $M$ to be to achieve a good accuracy? If we choose, for a given index $i_{p}, M=1$, then the wavefunction factorises into a simple product of contributions from the spaces $\left\{n_{1} \ldots n_{p}\right\}$ and $\left\{n_{p+1} \ldots n_{k}\right\}$. Increasing $M$ then introduces additional correlations or entanglement between the wavefunction components in the two spaces. The $M$ required for a given accuracy thus depends on the correlations in the specific state of the molecule. However, we have seen in our applications that for appropriate problems, even modest $M=O(100-1000)$ can allow us to obtain very good accuracy 
and to solve problems that are insoluble with other techniques. Of course, having a small number of variational parameters does not guarantee that an ansatz can be manipulated efficiently. (Witness the difficulty in evaluating the variational energy corresponding to a Coupled Cluster wavefunction!) As we shall see in Sec. 6. the product structure of the DMRG ansatz enables matrix elements to be evaluated without ever reconstructing the DMRG coefficients in the full Slater determinant expansion, thus bypassing the exponential complexity. (Although one can do so if one wishes, e.g. for the purposes of analysing the DMRG wavefunction, as in [14]). Finally, we note that the DMRG incorporates correlations between orbital spaces in a sequential manner, i.e. the first set of auxiliary indices $i_{1}$ entangles spaces $\left\{n_{1}\right\}$ and $\left\{n_{2} \ldots n_{k}\right\}, i_{2}$ entangles spaces $\left\{n_{1} n_{2}\right\}$ and $\left\{n_{3} \ldots n_{k}\right\}$ and so on. For this reason, the DMRG ansatz performs best if strongly-correlated orbitals are placed next to each other in the ansatz [12, 39, 42, 34].

5. A local multireference ansatz for long molecules: The DMRG wavefunction is particularly well-suited to long molecules where it can be viewed as a naturally local multireference ansatz. In long molecules (i.e. those where one of the dimensions is much larger than the other two) with a finite electronic correlation length, we can divide the molecule at any point along the backbone and expect the degree of entanglement between the two resulting subsystems to be independent of the point of division and the length of the chain. Thus, for such problems, the $M$ required for a given accuracy is independent of the length of the system and the number of variational parameters in the DMRG wavefunction is simply const $\times O(k)$, as should be in a local ansatz. However, unlike in other local correlation approaches the DMRG provides a local multireference ansatz. It is this local nature even in the presence of strong nondynamic correlations which has allowed us to solve very large active space multireference correlation problems in long molecules [7, 6, 5].

In problems which are large in two or three dimensions, the degree of entanglement between two subsystems grows exponentially with the length of the border, and thus the preceding considerations no longer apply. We might then ask, can we modify the DMRG ansatz to obtain a naturally local multireference description for large systems with arbitrary dimensionality? Recently, this has been shown to be possible. Consider, for example, two rows of atoms (each with one localised orbital) arranged as in Fig. 11. The first sub-figure illustrates the sequential coupling between orbital spaces that is contained in the DMRG wavefunction, which is inefficient at describing correlations between atoms in different rows. In the second sub-figure, however, we have added additional auxiliary indices to couple the site functions both along the rows as well as along the columns in a non-sequential manner. This is the basis for the so-called Pair-Entangled Product State wavefunctions which present one of the most promising new developments in this area [21, 22, 23]. 


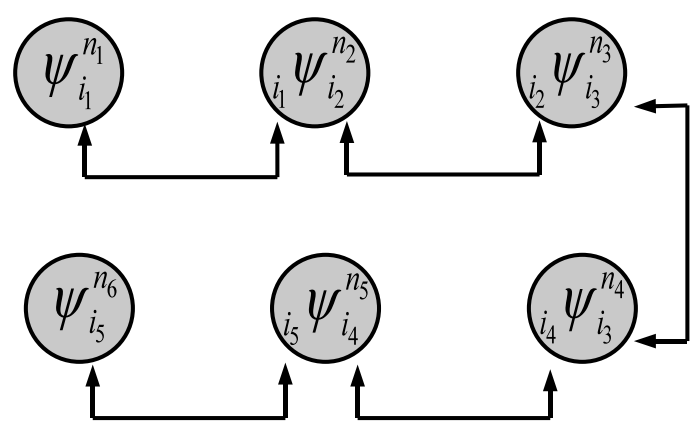

(a) DMRG

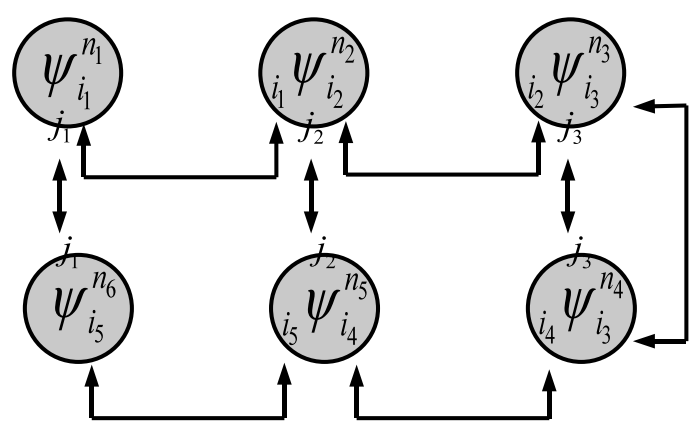

(b) PEPS

Fig. 1 Density Matrix Renormalisation Group and Pair Entangled Product State wavefunctions for two rows of atoms. Note in the DMRG ansatz, the site functions are coupled sequentially, which prevents the efficient description of correlations between the rows. However, in the PEPS ansatz, addition indices are added to the site functions (e.g. $\psi_{i_{1} i_{2}}^{n_{2}} \rightarrow \psi_{i_{1} i_{2} j_{2}}^{n_{2}}$ ) whose coupling directly captures the inter-row correlations.

\section{The Renormalized Basis}

As we have discussed above, the auxiliary indices of the site functions introduce couplings between the orbital spaces in the DMRG ansatz. In addition, they can also be provided with a direct physical interpretation. Just as the index $n_{i}$ is associated with the Fock space of orbital $i$, so can we also associate a set of renormalised manybody spaces with the auxiliary indices of each site function $\psi$. This provides the Renormalisation Group (RG) interpretation of the DMRG wavefunction. Consider, for example, the first set of auxiliary indices $i_{1}$. We first perform the summation in the DMRG wavefunction expression over $n_{1}$, which couples $\psi_{i_{1}}^{n_{1}}$ with the set of states $\left\{\left|n_{1}\right\rangle\right\}=\left\{|0\rangle,\left|1^{\alpha}\right\rangle,\left|1^{\beta}\right\rangle,\left|2^{\alpha \beta}\right\rangle\right\}$. This formally defines a space $\left\{i_{1}\right\}$ with basis functions $\left|i_{1}\right\rangle$

$$
\left|i_{1}\right\rangle=\sum_{n_{1}} \psi_{i_{1}}^{n_{1}}\left|n_{1}\right\rangle
$$


or more succinctly

$$
\left\{i_{1}\right\}=\hat{\psi}^{1} \cdot\left\{n_{1}\right\} .
$$

Of course, the transformation of the $\left\{n_{1}\right\}$ orbital Fock space by the $\psi^{1}$ site function is trivial. (Indeed, if, as is usual, we do not allow $\psi$ to mix states with different particle numbers or spin, we would simply have $\left|i_{1}\right\rangle=\left|n_{1}\right\rangle$ for all 4 states). However, things are more interesting, when we consider the spaces associated with later sets of auxiliary indices. For example, repeating the above exercise for $i_{2}$

$$
\begin{aligned}
\left|i_{2}\right\rangle & =\sum_{\substack{n_{1} n_{2} \\
i_{1}}} \psi_{i_{1}}^{n_{1}} \psi_{i_{1} i_{2}}^{n_{2}}\left|n_{1} n_{2}\right\rangle \\
& =\sum_{\substack{n_{2} \\
i_{1}}} \psi_{i_{1} i_{2}}^{n_{2}}\left|i_{1} n_{2}\right\rangle, \\
\left\{i_{2}\right\} & =\hat{\psi}^{2} \cdot\left\{i_{1} n_{2}\right\}=\hat{\psi}^{2} \cdot \hat{\psi}^{1} \cdot\left\{n_{1} n_{2}\right\} .
\end{aligned}
$$

In general for the space $\left\{i_{p}\right\}$ and the associated basis $\left|i_{p}\right\rangle$, we write

$$
\begin{aligned}
\left\{i_{p}\right\} & =\hat{\psi}^{p} \cdot\left\{i_{p-1} n_{p}\right\} \\
= & \hat{\psi}^{p} \cdot \hat{\psi}^{p-1} \ldots \hat{\psi}^{1} \cdot\left\{n_{1} n_{2} \ldots n_{p}\right\}, \\
\left|i_{p}\right\rangle= & \sum_{n_{p}} \psi_{i_{p-1} i_{p}}^{n_{p}}\left|i_{p-1} n_{p}\right\rangle \\
& i_{p-1} \\
= & \sum_{n_{1} \ldots n_{p}} \psi_{i_{1}}^{n_{1}} \psi_{i_{1} i_{2}}^{n_{2}} \ldots \psi_{i_{p-2} i_{p-1}}^{n_{p-1}} \psi_{i_{p-1} i_{p}}^{n_{p}}\left|n_{1} n_{2} \ldots n_{p}\right\rangle .
\end{aligned}
$$

Note that the matrix representation of $\hat{\psi}^{p}$ is simply the matrix form of the site function $\left[\psi^{p}\right]$ described in Eq. [11), i.e.

$$
\left\langle i_{p}\left|\hat{\psi}^{p}\right| i_{p-1} n_{p}\right\rangle=\psi_{i_{p}, i_{p-1} n_{1}}^{p}
$$

and thus we can also write Eq. (20) as

$$
\left|i_{p}\right\rangle=\sum_{n_{1} \ldots n_{p}}\left[\psi^{n_{1}}\right]\left[\psi^{n_{2}}\right] \ldots\left[\psi^{n_{p-1}}\right]\left[\psi^{n_{p}}\right]\left|n_{1} n_{2} \ldots n_{p}\right\rangle .
$$

Now the dimension of the $i_{p}$ index and $\left\{i_{p}\right\}$ space is fixed to be at most $M$ in the original ansatz (8). Thus, the action of $\hat{\psi}^{p} \ldots \hat{\psi}^{1}$ is a projective transformation from the full many-body space down into a renormalised many-body space of $M$ basis states, where each basis state $\left|i_{p}\right\rangle$ is expressed as a linear combination of many product functions $\left|n_{1} \ldots n_{p}\right\rangle$ with coefficients given by Eq. (20). The renormalised spaces have a recursive structure: $\left\{i_{p}\right\}$ is obtained from $\left\{i_{p-1}\right\}$ which is obtained from $\left\{i_{p-2}\right\}$ and so on.

The construction of one renormalised space from the previous one may be considered to proceed in two stages. To construct the space $\left\{i_{p}\right\}$, first we form the 


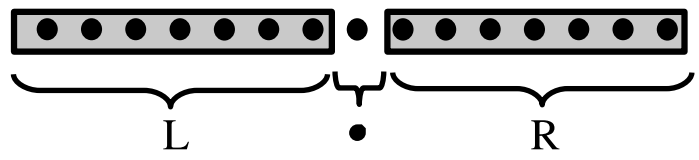

(a) One site ansatz

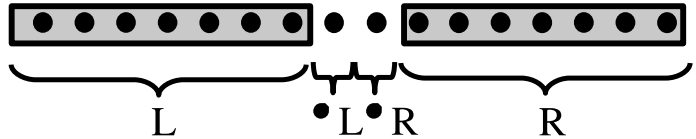

(b) Two site ansatz

Fig. 2 Block diagrams for the one and two site DMRG ansaetze.

product space

$$
\left\{i_{p-1}\right\} \otimes\left\{n_{p}\right\} \rightarrow\left\{i_{p-1} n_{p}\right\}
$$

and then we apply the projective transformation

$$
\hat{\psi}^{p} \cdot\left\{i_{p-1} n_{p}\right\} \rightarrow\left\{i_{p}\right\} .
$$

The first step is called "blocking" and the second step "decimation" in the traditional language of the Renormalisation Group, and therein lies the basic connection between the DMRG ansatz and its RG interpretation. It is common to represent these blocking and decimation steps in the pictorial fashion shown in Fig. 2.

\section{The Canonical Representation and Sweep algorithm}

The DMRG wavefunction is invariant to a class of transformations of the site functions $\psi$, since the associated nested many-body spaces $\left\{i_{p}\right\}$ are themselves invariant with respect to transformations within each space. The original DMRG algorithm, which was formulated in the language of orthogonal projective transformations following Wilson's Numerical Renormalisation Group, in fact corresponds to particular choices of representation of the site functions within the above invariant class. We shall call such representations "canonical representations". All existing DMRG implementations in quantum chemistry work with canonical representations of the DMRG wavefunction. In addition, the use of canonical representations is closely linked with the density matrix interpretation of the DMRG and also with the DMRG sweep algorithm, which provides a natural algorithm to optimise the DMRG wavefunction.

Associated with each DMRG wavefunction $\Psi$ there are $k$ canonical representations, one for each site. At site $p$, the canonical representation is written as 


$$
\begin{aligned}
|\Psi\rangle & =\sum_{n_{1} \ldots n_{p} \ldots n_{k}}\left[L^{n_{1}}\right] \ldots\left[L^{n_{p-1}}\right]\left[C^{n_{p}}\right]\left[R^{n_{p+1}}\right] \ldots\left[R^{n_{k}}\right]\left|n_{1} \ldots n_{p} \ldots n_{k}\right\rangle \\
& =\sum_{\substack{n_{1} \ldots n_{p} \ldots n_{k} \\
l_{1} \ldots l_{p-1}, r_{p} \ldots r_{k-1}}} L_{l_{1}}^{n_{1}} \ldots L_{l_{p-2} l_{p-1}}^{n_{p-1}} C_{l_{p-1} r_{p}}^{n_{p}} R_{r_{p} r_{p+1}}^{n_{p+1}} \ldots R_{r_{k-1}}^{n_{k}}\left|n_{1} \ldots n_{p} \ldots n_{k}\right\rangle .
\end{aligned}
$$

Here, the site functions to the left of $p$ have been given the symbol $L$, while those to the right have been given the symbol $R$. The $L$ and $R$ site functions, which are in this context usually called transformation matrices, are each orthogonal matrices when written in the matrix representation of Eq. (11). We interpret the $L$ site functions as matrices by grouping the $n$ index with the first auxiliary index,

$$
\left(q<p: L_{l n, l^{\prime}}^{q}:=L_{l l^{\prime}}^{n_{q}}\right)
$$

and in this form we have

$$
\begin{aligned}
{\left[L^{q}\right]^{T}\left[L^{q}\right] } & =[1], \\
\sum_{l n} L_{l n, l^{\prime}}^{q} L_{l n, l^{\prime \prime}}^{q} & =\delta_{l^{\prime} l^{\prime \prime}} .
\end{aligned}
$$

For the $R$ site functions, we group the $n$ index with the second auxiliary index

$$
\left(q>p: R_{r^{\prime}, r n}^{q}:=R_{r^{\prime} r}^{n_{q}}\right)
$$

and in this form we have

$$
\begin{aligned}
{\left[R^{q}\right]\left[R^{q}\right]^{T} } & =[1], \\
\sum_{r n} R_{r^{\prime}, r n}^{q} R_{r^{\prime \prime}, r n}^{q} & =\delta_{r^{\prime} r^{\prime \prime}} .
\end{aligned}
$$

The $L$ and $R$ matrices each define a set of orthogonal projective transformations, which give rise, respectively, to two sets of renormalised spaces $\{l\}$ and $\{r\}$ associated with the site $p$ representation of the DMRG wavefunction. The $\{l\}$ spaces, $\left\{l_{1}\right\},\left\{l_{2}\right\} \ldots$ are built up by incorporating the orbitals in the order $1,2 \ldots p$,

$$
\begin{aligned}
(q<p):\left\{l_{q}\right\} & =\hat{L}^{q} \cdot\left\{l_{q-1} n_{q}\right\} \\
& =\hat{L}^{q} \cdot \hat{L}^{q-1} \cdot\left\{l_{q-2} n_{q-1} n_{q}\right\} \\
& =\hat{L}^{q} \cdot \hat{L}^{q-1} \cdots \hat{L}^{1} \cdot\left\{n_{1} \ldots n_{q}\right\}
\end{aligned}
$$

and the $|l\rangle$ functions form an orthogonal renormalised basis (from the orthogonal nature of the $[L]$ transformation matrices) for each $\{l\}$ space

$$
\begin{aligned}
\left|l_{q}\right\rangle & =\sum_{n_{1} \ldots n_{p}}\left[L^{n_{1}}\right]\left[L^{n_{2}}\right] \ldots\left[L^{n_{q-1}}\right]\left[L^{n_{q}}\right]\left|n_{1} n_{2} \ldots n_{1}\right\rangle, \\
\left\langle l_{q} \mid l_{q}^{\prime}\right\rangle & =\delta_{l l^{\prime}} .
\end{aligned}
$$


The $\{r\}$ spaces and $|r\rangle$ basis functions are defined similarly, but now the orbitals are incorporated "backwards" in the order $k, k-1 \ldots p+1$

$$
\begin{aligned}
(q>p):\left\{r_{q}\right\} & =\hat{R}^{q} \cdot\left\{n_{q} r_{q+1}\right\} \\
& =\hat{R}^{q} \cdot \hat{R}^{q+1} \cdot\left\{n_{q} n_{q+1} r_{q+2}\right\} \\
& =\hat{R}^{q} \cdot \hat{R}^{q+1} \ldots \hat{R}^{k} \cdot\left\{n_{q} \ldots n_{k}\right\}, \\
\left|r_{q}\right\rangle & =\sum_{n_{q} \ldots n_{k}}\left[R^{n_{q}}\right]\left[R^{n_{q+1}}\right] \ldots\left[R^{n_{k-1}}\right]\left[R^{n_{k}}\right]\left|n_{q} n_{q+1} \ldots n_{k}\right\rangle, \\
\left\langle r_{q} \mid r_{q}^{\prime}\right\rangle & =\delta_{r r^{\prime}} .
\end{aligned}
$$

Having defined the renormalised spaces, we now see that the $C^{p}$ site function gives the wavefunction coefficients in the product space formed from the renormalised left basis $\left\{l_{p-1}\right\}$, the orbital space $\left\{n_{p}\right\}$, and the renormalised right basis $\left\{r_{p}\right\}$

$$
|\Psi\rangle=\sum_{l n r} C_{l n r}^{p}\left|l_{p-1} n_{p} r_{p}\right\rangle
$$

where we have used the notation $C_{l n r}^{p}:=C_{l_{p-1} r_{p}}^{n_{p}}$.

We now consider the DMRG wavefunction expressed in the canonical representations of sites other than $p$. Since the same wavefunction is simply being expressed in a different representation, this implies a relationship between the wavefunction coefficients $C$ and transformation matrices $L, R$ at different sites. Comparing representations at sites $p, p+1$ we see

$$
\begin{aligned}
|\Psi\rangle & =\sum_{n_{1} \ldots n_{p} \ldots n_{k}}\left[L^{n_{1}}\right] \ldots\left[L^{n_{p-1}}\right]\left[C^{n_{p}}\right]\left[R^{n_{p+1}}\right]\left[R^{n_{p+2}}\right] \ldots\left[R^{n_{k}}\right]\left|n_{1} \ldots n_{p} \ldots n_{k}\right\rangle \\
& =\sum_{n_{1} \ldots n_{p} \ldots n_{k}}\left[L^{n_{1}}\right] \ldots\left[L^{n_{p-1}}\right]\left[L^{n_{p}}\right]\left[C^{n_{p+1}}\right]\left[R^{n_{p+2}}\right] \ldots\left[R^{n_{k}}\right]\left|n_{1} \ldots n_{p} \ldots n_{k}\right\rangle .
\end{aligned}
$$

This implies

$$
\left[C^{n_{p}}\right]\left[R^{n_{p+1}}\right]=\left[L^{n_{p}}\right]\left[C^{n_{p+1}}\right]
$$

or, switching to the alternative matrix interpretation of Eq. (11) for $C^{p}, C^{p+1}$ and likewise for $L^{p}, R^{p+1}$

$$
\sum_{r} C_{l n, r}^{p} R_{r, r^{\prime} n}^{p+1}=\sum_{l^{\prime}} L_{l n, l^{\prime}}^{p} C_{l^{\prime}, n^{\prime} r^{\prime}}^{p+1}
$$

From $C^{p}$, we can determine the quantities in the site $p+1$ canonical form that do not explicitly appear in the site $p$ canonical form, namely $C^{p+1}, L^{p}$, by the singular value decomposition (SVD) of $C^{p}$, 

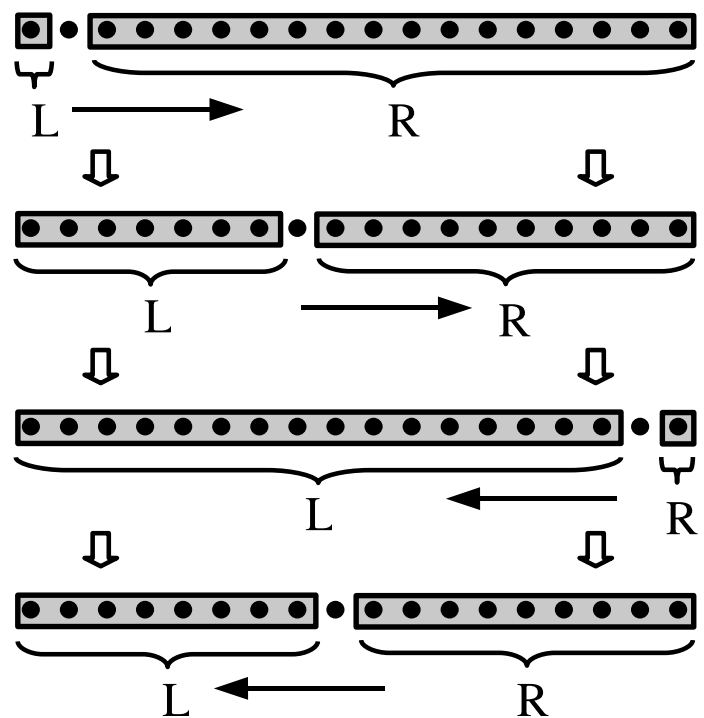

Fig. 3 The DMRG sweep algorithm with the one site ansatz. After all $L$ blocks are constructed going from $L \rightarrow R$, the sweep direction is reversed.

$$
\begin{gathered}
C_{l n, r}^{p}=\sum_{l^{\prime}} L_{l n, l^{\prime}}^{p} \sigma_{l^{\prime}} V_{l^{\prime} r}, \\
C_{l, n r}^{p+1}=\sum_{r^{\prime}} \sigma_{l} V_{l r^{\prime}} R_{r^{\prime}, r n}^{p+1} .
\end{gathered}
$$

The connection through the SVD between the representations at different sites leads to the density matrix formulation of the DMRG. Recall that the singular vectors of a matrix $M$ may be related to the eigenvectors of $M^{T} M$ and $M M^{T}$. Thus from $C^{p}$, we can define a symmetric object $\left[\Gamma^{p}\right]=\left[C^{p}\right]^{T}\left[C^{p}\right]$, i.e.

$$
\Gamma_{l n, l^{\prime} n^{\prime}}^{p}=\sum_{r} C_{l n r}^{p} C_{l^{\prime} n^{\prime} r}^{p}
$$

$\Gamma^{p}$ is none other than the density matrix associated with the left subsystem, or "block" of orbitals $1 \ldots p$, and the left transformation matrix $L^{p}$ is obtained as the matrix of $M$ eigenvectors

$$
\sum_{l^{\prime} n^{\prime}} \Gamma_{l n, l^{\prime} n^{\prime}}^{p} L_{l^{\prime} n^{\prime}, l^{\prime \prime}}^{p}=L_{l n, l^{\prime \prime}}^{p} \sigma_{l^{\prime \prime}}^{2}
$$

This corresponds to the traditional density matrix interpretation of the DMRG: to obtain the canonical representation at a new site requires a basis change into the eigenvectors of the subsystem density matrix.

The sequential set of transformations from representation to representation along the sites also yields a natural optimisation procedure for the DMRG wavefunction known as the sweep algorithm. At each site $p$, we solve the Schrödinger equation 
in the basis $\left\{l_{p-1} n_{p} r_{p}\right\}$ to obtain the coefficient matrix $C^{p}$, thus (dropping the subscripts on the basis functions for simplicity)

$$
\begin{aligned}
\left\langle l^{\prime} n^{\prime} r^{\prime}|\hat{H}-E| \Psi\right\rangle & =0 \\
\sum_{l n r}\left\langle l^{\prime} n^{\prime} r^{\prime}|\hat{H}-E| l n r\right\rangle C_{l n r}^{p} & =0
\end{aligned}
$$

From this coefficient matrix, we obtain the new transformation matrix at site $p \pm 1$ from the SVD in Eq. (44) (or equivalently, in the density matrix formulation, from the eigenvectors of the density matrix in Eq. (47)). If we move through the sites from left to right $(p \rightarrow p+1)$ in a sweep, we successively determine new $L^{p}$ matrices, while moving from right to left ( $p \rightarrow p-1)$ determines new $R^{p}$ matrices. After the sites are traversed in one direction, we traverse in the opposite direction thus allowing improvement of all the $L^{p}$ and $R^{p}$ matrices. (Of course, to initialise the procedure, requires some starting guess for the $L^{p}$ and $R^{p}$ matrices). This is the basic method that is employed to optimise the DMRG energy.

We usually depict the canonical representation at site $p$ in a block-configuration diagram as shown in Fig. 2 consisting of a left block of orbitals $1 \ldots p-1$, the site $p$ and a right block of orbitals $p+1 \ldots k$. Then, moving from one site to another corresponds to moving from block-configuration to block-configuration, sweeping from left-to-right and then right-to-left as shown in Fig. 3.

So far we have always been working within what is known as the one-site DMRG algorithm, since, as can be seen from the block diagram in Fig. 22 there is only one site between the left and right blocks. However, in earlier formulations of the DMRG algorithm it was common to use the so-called two-site algorithm, corresponding to the second block configuration in Fig. 2. Here the wavefunction at site $p$ is written in the renormalised product space as

$$
|\Psi\rangle=\sum_{l n n^{\prime} r} C_{l n n^{\prime} r}^{p}\left|l_{p-1} n_{p} n_{p+1}^{\prime} r_{p+1}\right\rangle
$$

where we see that two complete orbital Fock spaces $\left\{n_{p}\right\},\left\{n_{p+1}\right\}$ appear in the wavefunction expansion. Unlike in the one-site configuration, we can only approximately relate the canonical representations of the two-site wavefunctions at different sites, and thus there is no single consistent DMRG wavefunction across a two-site DMRG sweep, but rather a whole family of DMRG wavefunctions, one at each site. Originally, the two-site algorithm was introduced to eliminate some numerical problems associated with local minima when optimising the DMRG wavefunction in the sweep algorithm [2, 12, 37], but with the introduction of newer methods which avoid such minima [56], the one-site formulation should now be viewed as preferred. 


\section{Evaluation of matrix elements}

For completeness, we now outline briefly how the DMRG wavefunction allows the efficient evaluation of the matrix elements necessary to solve the Schrodinger equation in the renormalised product basis (49). We first note that any operator in the complete Fock space $\left\{n_{1}\right\} \otimes\left\{n_{2}\right\} \otimes\left\{n_{3}\right\} \otimes \ldots \otimes\left\{n_{k}\right\}$ can be expressed as a sum of products of "local" operators that each act individually in the Fock space of a single site. For example, the unit operator $\hat{I}$ in the full Fock space may be considered as a single tensor product of local unit operators

$$
\hat{I}=\hat{I}^{1} \otimes \hat{I}^{2} \otimes \hat{I}^{3} \otimes \ldots \otimes \hat{I}^{k}
$$

where e.g. $\left\langle n_{1}\left|\hat{I}^{1}\right| n_{1}\right\rangle=\delta_{n_{1} n_{1}^{\prime}}$. To see how the quantum chemistry Hamiltonian

$$
H=\sum_{i j} t_{i j} a_{i}^{\dagger} a_{j}+\sum_{i j k l} v_{i j k l} a_{i}^{\dagger} a_{j}^{\dagger} a_{k} a_{l}
$$

can be written as a sum of products of local operators, it is sufficient to show that the creation and annihilation operators can be expressed in this form. Note that a single creation or annihilation operator does not simply act in the Fock space of a single orbital, because of the anticommutation relations between operators. Instead, we write for $a_{i}^{\dagger}, a_{i}$

$$
\begin{aligned}
a_{i}^{\dagger} & =\prod_{j<i}(-)^{n_{j}} \otimes P_{i} a_{i}^{\dagger} P_{i}, \\
a_{i} & =\prod_{j<i}(-)^{n_{j}} \otimes P_{i} a_{i} P_{i} .
\end{aligned}
$$

Here the operator $\prod_{j<i}(-)^{n_{j}}$ formally keeps tracks of the anticommutation, since if we consider e.g. $a_{i}$ acting on a determinant, it counts the number of sign changes involved in moving orbital $i$ to the front of the orbital string. $P_{i}$ denotes the projection of operator onto the $\left\{n_{i}\right\}$ space alone.

Given that all operators can be written as a sum of products of local operators, we now examine how the matrix elements of a single product of local operators are obtained. Consider the product

$$
\hat{O}=\hat{O}^{1} \otimes \hat{O}^{2} \otimes \ldots \hat{O}^{k}
$$

In terms of the product basis $\left\{\left|l_{p_{1}} n_{p} r_{p}\right\rangle\right\}$ of site $p$, we can write (dropping the subscripts on the basis functions for simplicity)

$$
\begin{aligned}
\left\langle l^{\prime} n^{\prime} r^{\prime}|\hat{O}| \ln r\right\rangle & =\left\langle l^{\prime}\left|\hat{O}^{1} \otimes \ldots \otimes \hat{O}^{p-1}\right| l\right\rangle\left\langle n^{\prime}\left|\hat{O}^{p}\right| n\right\rangle\left\langle r^{\prime}\left|\hat{O}^{p} \otimes \ldots \otimes \hat{O}^{k}\right| r\right\rangle \\
& =\left\langle l^{\prime}\left|\hat{O}_{L}\right| l\right\rangle\left\langle n^{\prime}\left|\hat{O}^{p}\right| n\right\rangle\left\langle r^{\prime}\left|\hat{O}_{R}\right| r\right\rangle .
\end{aligned}
$$

It is sufficient to demonstrate how the matrix elements $\left\langle l\left|\hat{O}_{L}\right| l^{\prime}\right\rangle$ are calculated as those for $O_{R}$ are obtained in a similar manner. From the recursive definitions of the 
renormalised basis functions $|l\rangle,\left|l^{\prime}\right\rangle$ in Eqs. (34), (37) we have

$$
\left\langle l^{\prime}\left|\hat{O}_{L}\right| l\right\rangle=\sum_{\substack{n_{1} \ldots n_{p} \\ n_{1}^{\prime} \ldots n_{p}^{\prime}}}\left[L^{n_{1}}\right]\left[L^{n_{2}}\right] \ldots\left[L^{n_{p-1}}\right]\left(O_{n_{1} n_{1}^{\prime}}^{1} O_{n_{2} n_{2}^{\prime}}^{2} \ldots O_{n_{p-1} n_{p-1}^{\prime}}^{p-1}\right)\left[L^{n_{1}^{\prime}}\right]\left[L^{n_{2}^{\prime}}\right] \ldots\left[L^{n_{p-1}^{\prime}}\right] .
$$

These multiple transformations may be efficiently organised into groups of two step procedures (corresponding to the familiar blocking and decimation steps of the RG). Writing

$$
O_{l_{1} l_{1}^{\prime}}^{1}=\left\langle l_{1}\left|\hat{O}_{1}\right| l_{1}^{\prime}\right\rangle=L_{l_{1}}^{n_{1}} O_{n_{1} n_{1}^{\prime}}^{1} L_{l_{1}}^{n_{1}}
$$

the blocking step corresponds to

$$
O_{l_{1} l_{1}^{\prime}}^{1} \otimes O_{n_{2} n_{2}^{\prime}}^{2} \rightarrow\left(O^{1} O^{2}\right)_{l_{1} n_{1} l_{1}^{\prime} n_{1}^{\prime}}
$$

while the decimation corresponds to the transformation into the renormalised basis $\left(\left\{l_{1} n_{2}\right\} \rightarrow\left\{l_{2}\right\}\right)$

$$
\sum_{l_{1} n_{2} l_{1}^{\prime} n_{2}^{\prime}} L_{l_{1} l_{2}}^{n_{2}}\left(O^{1} O^{2}\right)_{l_{1} n_{2} l_{1}^{\prime} n_{2}^{\prime}} L_{l_{1}^{\prime} l_{2}^{\prime}}^{n_{2}^{\prime}} \rightarrow\left(O^{1} O^{2}\right)_{l_{2} l_{2}^{\prime}}
$$

Each such transformation has the cost of a matrix multiplication i.e $O\left(M^{3}\right)$, and because of the recursive structure of the transformations, the complete matrix element $\left\langle l^{\prime}\left|O_{L}\right| l\right\rangle$ may be efficiently evaluated as a sequence of matrix products with a total cost $O\left(M^{3} k\right)$.

For complicated operators such as the quantum chemical Hamiltonian which consist of sums over many products of operators, it is clear that there are intermediates which can be reused and saved. For example, the matrix elements of $a_{1}^{\dagger} a_{2}^{\dagger} a_{9} a_{10}$ and $a_{1}^{\dagger} a_{2}^{\dagger} a_{4} a_{5}$ both involve as an intermediate the renormalised representation of $a_{1}^{\dagger} a_{2}^{\dagger}$, which may be stored and reused. In practice, therefore, the optimal implementation of the DMRG algorithm in quantum chemistry requires an efficient organisation of intermediates and this is primarily where most of the complexity may be be found. The interested reader is referred to the literature for further details e.g. [10, 11, 12, 13, 14, 37].

\section{Conclusions}

In this article we have attempted to introduce the Density Matrix Renormalisation Group (DMRG) primarily from the view that it provides quantum chemistry with a new kind of wavefunction ansatz. Consequently, we can analyse and manipulate the ansatz in the way to which we are accustomed in quantum chemistry. By ex- 
amining its structure we arrive at an intuitive understanding of the strengths of the DMRG method e.g. in multireference problems, or in long molecules, where it is a naturally local multireference approach. A striking feature of the DMRG ansatz as compared to other quantum chemical wavefunctions is the recursive structure. This is the connection between the DMRG wavefunction and the traditional language of the Renormalisation Group, and provides the central mechanism behind the efficient evaluation of matrix elements in the method.

Traditionally quantum chemistry has understood electronic structure in terms of the many-electron wavefunction. We hope that by thinking about the DMRG in this language, it will not only become more accessible, but new possibilities will arise for cross-fertilisation between quantum chemical techniques and the Density Matrix Renormalisation Group.

\section{Acknowledgments}

Garnet Kin-Lic Chan would like to acknowledge support from Cornell University, the Cornell Center for Materials Research (CCMR), the David and Lucile Packard Foundation, the National Science Foundation CAREER program CHE-0645380, the Alfred P. Sloan Foundation, and the Department of Energy, Office of Science through award DE-FG02-07ER46432. Johannes Hachmann would like to acknowledge support provided by a Kekulé Fellowship of the Fond der Chemischen Industrie. Eric Neuscamman would like to acknowledge support provided by a National Science Foundation Graduate Research Fellowship.

\section{References}

1. S.R. White, Phys. Rev. Lett. 69(19), 2863 (1992)

2. S.R. White, Phys. Rev. B 48(14), 10345 (1993)

3. G.K.L. Chan, M. Head-Gordon, J. Chem. Phys. 118(19), 8551 (2003)

4. G.K.L. Chan, M. Kállay, J. Gauss, J. Chem. Phys. 121(13), 6110 (2004)

5. J. Hachmann, J.J. Dorando, M. Avilés, G.K.L. Chan, J. Chem. Phys. 127(13), 134309 (2007)

6. J.J. Dorando, J. Hachmann, G.K.L. Chan, J. Chem. Phys. 127(8), 084109 (2007)

7. J. Hachmann, W. Cardoen, G.K.L. Chan, J. Chem. Phys. 125(14), 144101 (2006)

8. K.G. Wilson, Rev. Mod. Phys. 47(4), 773 (1975)

9. K.G. Wilson, Rev. Mod. Phys. 55(3), 583 (1983)

10. S.R. White, R.L. Martin, J. Chem. Phys. 110(9), 4127 (1999)

11. A.O. Mitrushenkov, G. Fano, F. Ortolani, R. Linguerri, P. Palmieri, J. Chem. Phys. 115(15), 6815 (2001)

12. G.K.L. Chan, M. Head-Gordon, J. Chem. Phys. 116(11), 4462 (2002)

13. Ö. Legeza, J. Röder, B.A. Hess, Phys. Rev. B 67(12), 125114 (2003)

14. G. Moritz, M. Reiher, J. Chem. Phys. 126(24), 244109 (2007)

15. M. Fannes, B. Nachtergaele, R.F. Werner, Comm. Math. Phys. 144(3), 443 (1992)

16. M. Fannes, B. Nachtergaele, R.F. Werner, J. Funct. Anal. 120(2), 511 (1994)

17. S. Östlund, S. Rommer, Phys. Rev. Lett. 75(19), 3537 (1995)

18. S. Rommer, S. Östlund, Phys. Rev. B 55(4), 2164 (1997) 
19. F. Verstraete, J.J. García-Ripoll, J.I. Cirac, Phys. Rev. Lett. 93(20), 207204 (2004)

20. F. Verstraete, D. Porras, J.I. Cirac, Phys. Rev. Lett. 93(22), 227205 (2004)

21. F. Verstraete, J.I. Cirac, arXiv:cond-mat 0407066v1 (2004)

22. D. Pérez-Garciá, F. Verstraete, J.I. Cirac, M.M. Wolf, arXiv:quant-ph 0707.2260v1 (2007)

23. N. Schuch, M.M. Wolf, F. Verstraete, J.I. Cirac, Phys. Rev. Lett. 98(14), 140506 (2007)

24. V. Murg, F. Verstraete, J.I. Cirac, Phys. Rev. A 75(3), 033605 (2007)

25. F. Verstraete, A. Weichselbaum, U. Schollwöck, J.I. Cirac, J. von Delft, arXiv:cond-mat 0504305v1 (2005)

26. S.R. White, A.E. Feiguin, Phys. Rev. Lett. 93(7), 076401 (2004)

27. A.J. Daley, C. Kollath, U. Schollwöck, G. Vidal, J. Stat. Mech.: Theor. Exp. (04), P04005 (2004)

28. G. Vidal, Phys. Rev. Lett. 93(4), 040502 (2004)

29. G. Vidal, arXiv:quant-ph 0610099v1 (2006)

30. K. Hallberg, in Theoretical Methods for Strongly Correlated Electrons, ed. by D. Sénéchal, A.M. Tremblay, C. Bourbonnais, CRM Series in Mathematical Physics (Springer, New York, 2003)

31. K.A. Hallberg, Adv. Phys. 55(5), 477 (2006)

32. U. Schollwöck, Rev. Mod. Phys. 77(1), 259 (2005)

33. S. Daul, I. Ciofini, C. Daul, S.R. White, Int. J. Quantum Chem. 79(6), 331 (2000)

34. J. Rissler, R.M. Noack, S.R. White, Chem. Phys. 323(2-3), 519 (2006)

35. A.O. Mitrushenkov, R. Linguerri, P. Palmieri, G. Fano, J. Chem. Phys. 119(8), 4148 (2003)

36. A.O. Mitrushenkov, G. Fano, R. Linguerri, P. Palmieri, arXiv:cond-mat 0306058v1 (2003)

37. G.K.L. Chan, J. Chem. Phys. 120(7), 3172 (2004)

38. G.K.L. Chan, T. Van Voorhis, J. Chem. Phys. 122(20), 204101 (2005)

39. Ö. Legeza, J. Sólyom, Phys. Rev. B 68(19), 195116 (2003)

40. Ö. Legeza, J. Röder, B.A. Hess, Mol. Phys. 101(13), 2019 (2003)

41. Ö. Legeza, J. Sólyom, Phys. Rev. B 70(20), 205118 (2004)

42. G. Moritz, B.A. Hess, M. Reiher, J. Chem. Phys. 122(2), 024107 (2005)

43. G. Moritz, A. Wolf, M. Reiher, J. Chem. Phys. 123(18), 184105 (2005)

44. G. Moritz, M. Reiher, J. Chem. Phys. 124(3), 034103 (2006)

45. D. Zgid, M. Nooijen, J. Chem. Phys. In print

46. S. Ramasesha, S.K. Pati, H.R. Krishnamurthy, Z. Shuai, J.L. Brédas, Synth. Met. 85(1-3), $1019(1997)$

47. D. Yaron, E.E. Moore, Z. Shuai, J.L. Brédas, J. Chem. Phys. 108(17), 7451 (1998)

48. Z. Shuai, J.L. Brédas, A. Saxena, A.R. Bishop, J. Chem. Phys. 109(6), 2549 (1998)

49. G. Fano, F. Ortolani, L. Ziosi, J. Chem. Phys. 108(22), 9246 (1998)

50. G.L. Bendazzoli, S. Evangelisti, G. Fano, F. Ortolani, L. Ziosi, J. Chem. Phys. 110(2), 1277 (1999)

51. C. Raghu, Y. Anusooya Pati, S. Ramasesha, Phys. Rev. B 65(15), 155204 (2002)

52. C. Raghu, Y. Anusooya Pati, S. Ramasesha, Phys. Rev. B 66(3), 035116 (2002)

53. J. Dukelsky, M.A. Martín-Delgado, T. Nishino, G. Sierra, Europhys. Lett. 43(4), 457 (1998)

54. F. Verstraete, J.I. Cirac, Phys. Rev. B 73(9), 094423 (2006)

55. D. Pérez-Garciá, F. Verstraete, M.M. Wolf, J.I. Cirac, Quant. Inf. Comp. 7(5\&6), 401 (2007)

56. S.R. White, Phys. Rev. B 72(18), 180403 (2005) 